\title{
Multicentric Glioblastoma Multiforme Mimicking Optic Neuritis
}

\author{
Francesco Pellegrini ${ }^{\mathrm{a}}$, Andrew G. Lee ${ }^{\mathrm{b}, \mathrm{c}, \mathrm{d}, \mathrm{e}, \mathrm{f}, \mathrm{g}, \mathrm{h}}$, and Cristina Cercato ${ }^{\mathrm{i}}$

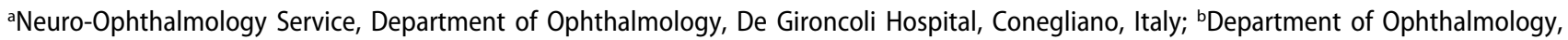 \\ Blanton Eye Institute, Houston Methodist Hospital, Houston, Texas, USA; 'Baylor College of Medicine, Houston, Texas, USA; ${ }^{\mathrm{D} D e p a r t m e n t s ~ o f ~}$ \\ Ophthalmology, Neurology, and Neurosurgery, Weill Cornell Medicine, New York, New York, USA; 'Department of Ophthalmology, The \\ University of Texas Medical Branch, Galveston, Texas, USA; fDepartment of Ophthalmology, The University of lowa Hospitals and Clinics,

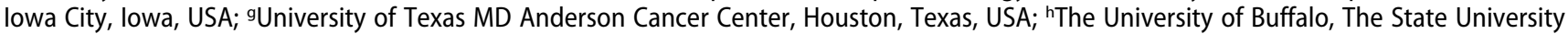 \\ of New York, Buffalo, New York, USA; 'Department of Radiology, Vittorio Veneto Hospital, Vittorio Veneto, Italy
}

\section{ABSTRACT}

A 49-year-old previously healthy woman presented with acute painless visual loss in the right eye, a right relative afferent pupillary defect, and a normal fundus examination. She was diagnosed with retrobulbar "optic neuritis" and given a course of intravenous steroids. Despite treatment, however, she continued to lose vision and serial visual field testing confirmed a junctional scotoma in the fellow eye. Cranial magnetic resonance imaging (MRI) showed a mass at the junction between the right optic nerve and the anterior aspect of the chiasm and a right frontal lesion that proved to be multicentric glioblastoma multiforme. Clinicians should be aware of the possibility of aggressive neoplasm in the differential diagnosis of retrobulbar optic neuritis.
ARTICLE HISTORY

Received 2 June 2017

Revised 17 June 2017

Accepted 29 June 2017

\section{KEYWORDS}

Glioblastoma multiforme; malignant optic nerve glioma of the adult; optic nerve tumour; optic neuritis

\section{Case report}

A 49-year-old woman presented to her ophthalmologist complaining of new onset, painless, decreased vision in the right eye (OD) for 2 days. Best-corrected visual acuity (BCVA) was 20/40 OD and 20/20 in the left eye (OS). There was a right relative afferent pupillary defect (RAPD). Slit-lamp biomicroscopy, external, motility, intraocular pressure, and fundus examinations were normal in both eyes (OU). Automated visual field testing disclosed diffuse depression OD and a mild superotemporal depression OS (Figure 1). She was diagnosed with retrobulbar "optic neuritis" OD and given a course of 3 days of intravenous (IV) $1000 \mathrm{mg}$ of methylprednisolone followed by an oral taper. After 10 days, however, she returned to the emergency department complaining of progressive visual loss OD despite steroid treatment.

Neuro-ophthalmological examination showed a BCVA of hand movement OD and 20/20 OS. There was a large right RAPD. Repeat automated visual field confirmed a superotemporal field depression (i.e., junctional scotoma) OS suggestive of posterior right optic nerve/anterior chiasm involvement (Figure 2). The remainder of the eye examination was normal OU. Magnetic resonance imaging (MRI) of the brain with and without contrast showed a right frontal lobe mass with contrast enhancement and a second mass with ring enhancement located at the anterior aspect of the chiasm, on the right (Figure 3). Subsequent brain biopsy of the frontal lobe lesion was consistent with a glioblastoma multiforme (GBM).

\section{Discussion}

Our case illustrates three major clinical points. First, although the most common cause of an acute unilateral retrobulbar optic neuropathy in a young to middle-aged adult is retrobulbar optic neuritis, other optic neuropathies should be considered in the differential diagnosis. The differential diagnosis is based on many factors, including the time course of the visual loss (e.g., acute, subacute, or chronic), the ophthalmoscopic appearance of the optic nerve

CONTACT Francesco Pellegrini francepellegrini@virgilio.it Department of Ophthalmology, De Gironcoli Hospital, Via D. Manin 110, 31015 Conegliano (TV), Italy. 

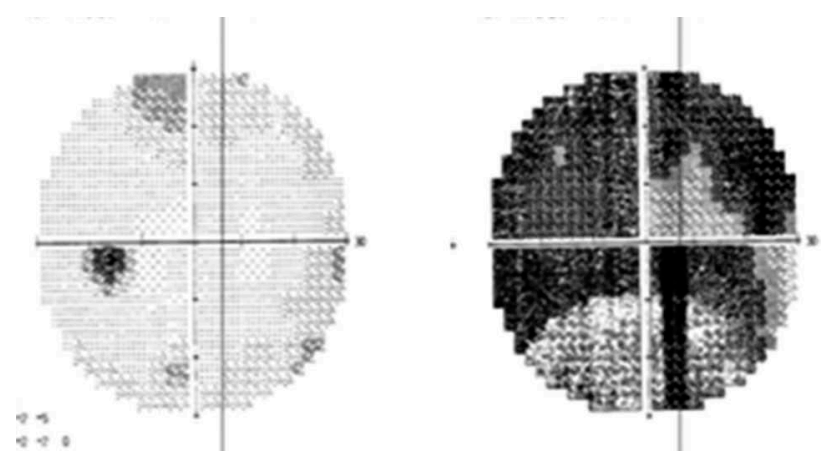

Figure 1. Automated visual field at presentation demonstrating diffuse depression $\mathrm{OD}$ and a mild superotemporal depression OS consistent with the junctional scotoma.
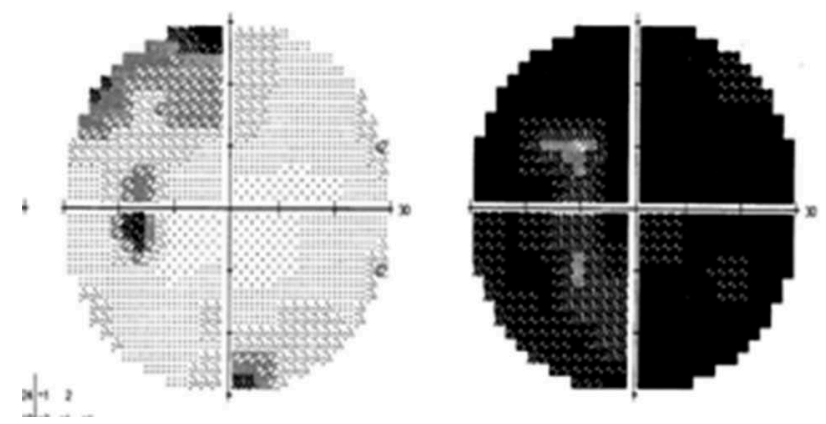

Figure 2. Repeat automated visual field confirms the junctional scotoma OS suggestive of posterior right optic nerve/anterior chiasm involvement.

(e.g., normal, pale, or swollen); the patient age (e.g., younger in optic neuritis and older in ischaemic optic neuropathy) and sex; the presence of other symptoms (e.g., pain with eye movement) or systemic symptoms; and the course (e.g., steroid responsive/nonresponsive or steroid dependent). The typical presentation for optic neuritis (ON) is a young female with acute loss of vision, evidence for an optic neuropathy (e.g., loss of visual acuity and visual field defect associated to a RAPD), pain with eye movement, and a normal fundus. Idiopathic and demyelinating $\mathrm{ON}$ are the most common aetiologies for $\mathrm{ON}$ in adults.

Contrast brain and orbital MRI with fat suppression is typically performed to look for the diagnostic radiographic features of $\mathrm{ON}$, including contrast enhancement or T2-weighted imaging abnormalities of the ipsilateral optic nerve and in the case of demyelinating disease (e.g., multiple sclerosis [MS]), periventricular white matter lesions. The MRI is useful for confirming the diagnosis of retrobulbar $\mathrm{ON}$ and for prognostic

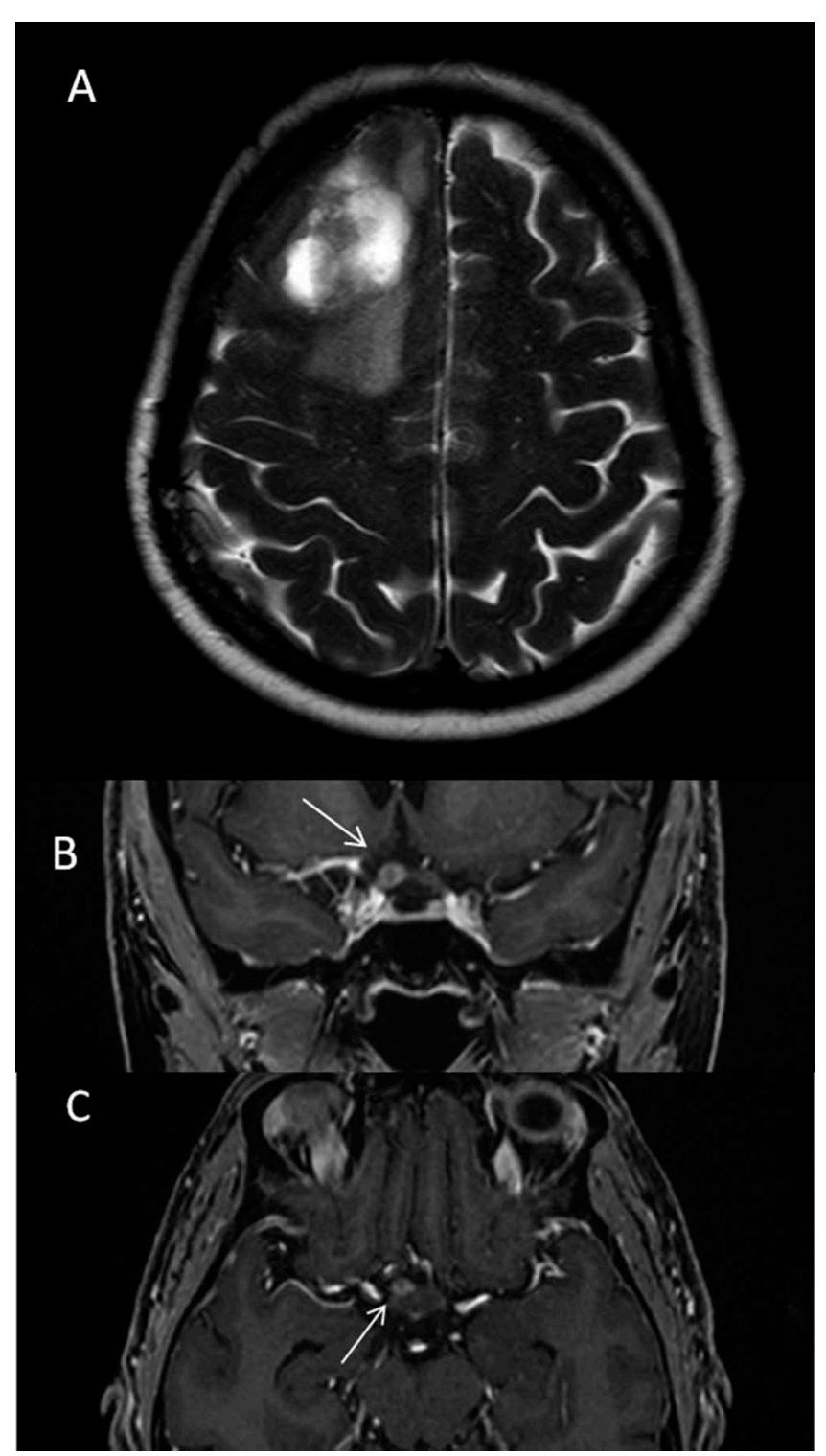

Figure 3. Brain MRI. (A) Axial T2-weighted image shows a heterogeneous multilobulated hyperintense mass in the right frontal lobe with adjacent vasogenic oedema and mild displacement of the midline due to mass effect. (B) Coronal and (C) axial T1-weighted images of a second mass (arrows) located at the anterior aspect of the chiasm, on the right, with ring enhancement due to neoplastic infiltration.

information about the future development of clinically definite MS. In the Optic Neuritis Treatment Trial (ONTT), MRI showed two compressive lesions mimicking retrobulbar ON (pituitary adenoma and ophthalmic artery aneurysm). ${ }^{1}$ Although the absence of pain and age older than 45 do not exclude demyelinating optic neuritis, the occurrence of acute painless retrobulbar $\mathrm{ON}$ in an older patient should prompt consideration for other aetiologies. 
Second, the pattern of visual field loss can be helpful in the differential diagnosis. Typically, an ipsilateral nerve fibre layer defect (e.g., arcuate, altitudinal) or central scotoma occurs in ON. Although contralateral visual field defects are seen in patients with $\mathrm{ON}$, the presence of a superotemporal visual field defect in the contralateral eye is a special form of localising defect (i.e., the junctional scotoma).

Wilbrand and Saenger ${ }^{2,3}$ studied the anatomy of the optic chiasm after enucleation and noted that inferonasal crossing fibres curved anteriorly into the contralateral optic nerve for 1-2 $\mathrm{mm}$, before bending back into the chiasm and then into the optic tract. This anatomical finding was known as the "Wilbrand knee" and was invoked to explain the contralateral superotemporal visual field defect when the optic nerve is damaged at the chiasmal junction. About 90 years later, Horton ${ }^{4}$ argued that the Wilbrand knee may be an enucleation artefact. In his detail anatomical studies on primates and human cadavers, he argued that the Wilbrand knee might be the result of loss of myelin and atrophy of the remaining portion of the optic nerve proximal to transaction. He noted that the Wilbrand knee was absent up to a few months after enucleation or transaction of the nerve, but developed after long-term enucleation, and concluded that the Wilbrand knee does not exist in the normal primate optic chiasm and may occur as an artefact from the shrinkage of the optic chiasm and atrophy of fibres from the enucleated eye. In contrast, Shin et al. ${ }^{5}$ were able to demonstrate the Wilbrand knee. These authors made thin $(25 \mu \mathrm{m})$ sections of the chiasm that were illuminated and digitally imaged from different angles. They used the anisotropic light-reflecting properties of myelinated axons in the human chiasm, and each of the images was reproduced in a pseudo-colour map in red, green, or blue. Whereas superior chiasm sections showed no curving of crossing fibres, in the inferior chiasm sections there was a sheet of crossing fibres originating from the anterior chiasm and bending towards the contralateral optic nerve before arching back towards the optic tract, consistent with the original descriptions of Wilbrand and Saenger. Regardless of the anatomical existence of the Willbrand knee, however, the clinical localising value to the junction in a patient with an ipsilateral optic neuropathy and a contralateral superotemporal visual field defect remains unchanged.

Interestingly, Traquair, a Scottish ophthalmologist, first used the term "junction scotoma" to refer to a temporal monocular hemianopic scotoma produced by damage to nasal fibres of the intracranial optic nerve at its junction with the optic chiasm. ${ }^{6,7}$ Thus, the term was used when a strictly unilateral temporal hemicentral visual field defect was present. Unfortunately there is continuing confusion regarding the term "junctional scotoma".

It has become common practice to use the term "junctional scotoma" to refer not to the field defect in the affected eye but rather to the superior temporal field defect seen in the opposite unaffected eye. Miller ${ }^{6,8}$ recommended that the unilateral temporal visual field defect described by Traquair should be referred to as the "junctional scotoma of Traquair" whereas the term "junctional scotoma" should be used to describe a contralateral superotemporal visual field defect. ${ }^{9}$

A compressive lesion should be suspected in either form of junctional visual field loss., ${ }^{9,10}$ Hickman et al. ${ }^{11}$ retrospectively studied 135 patients with acute $\mathrm{ON}$ and found that nine patients with abnormal MRI enhancement of the intracranial optic nerve immediately anterior to, but not including, the optic chiasm. Of these cases, two exhibited a superotemporal visual field defect in the contralateral eye (i.e., the junctional scotoma). They concluded that an acute lesion of the posterior optic nerve in $\mathrm{ON}$ can occasionally give rise to a superotemporal visual field depression in the asymptomatic fellow eye. Therefore, the occurrence of a contralateral superotemporal visual field defect does not exclude typical ON from the list of the possible aetiologies.

Third, other clinical features in our case were atypical for $\mathrm{ON}$ (e.g., older age, lack of pain with eye movement, and progression of visual loss despite steroid treatment); atypical features should direct the physician toward an alternative diagnosis.

In adults with multicentric glioblastoma multiforme (GBM), the frontal lobes are most often involved, followed by the temporal lobes. ${ }^{12}$ Less than $20 \%$ of GBMs are multifocal or multicentric. 
Whereas benign optic nerve glioma is the most common optic nerve tumour (especially in children), malignant optic glioma of the adult (MOGA) is rare and was first defined by Hoyt et al. in 1973. ${ }^{13}$ MOGA is a high-grade glioma typically presenting as an anaplastic astrocytoma (grade III) or glioblastoma (grade IV). Mean age at diagnosis is 54 years. At onset, up to $70 \%$ of cases present with unilateral decreased vision, $41 \%$ had optic disc oedema, and interestingly pain is present in $20 \%$ of cases. These findings can mimic optic neuritis. The visual loss from MOGA inexorably progresses, and the mean interval from initial symptoms to total blindness is $3.3 \pm 2.8$ months. ${ }^{14}$ The neuroimaging appearance of MOGA is not specific for neoplasm, but the optic nerve may be thickened and may show enhancement with heterogeneous and cystic areas. ${ }^{15}$ The prognosis for patients with MOGA is poor with the median survival of approximately 12 months. ${ }^{16}$ At presentation, MOGA may mimic either optic neuritis or ischaemic optic neuropathy, but the history, clinical examination, and paraclinical testing can help differentiate these entities from one another. ${ }^{16}$ Clinically speaking, MOGA may produce one of two clinical pictures. ${ }^{17,18}$ If the tumour is posterior (intracranial optic nerve or chiasm), there is rapidly progressive visual loss associated with optic disc atrophy. However, the optic nerve may initially appear normal and may be initially mistaken for retrobulbar ON particularly when there is associated pain. When the tumour initially affects the proximal portion of the intraorbital optic nerve, there is acute/subacute loss of vision associated with optic disc swelling.

There are few prior reports in the English language ophthalmic literature of MOGA simulating an acute $\mathrm{ON},{ }^{19,20}$ but in most of these cases the optic nerve was swollen at presentation. In a recent series of five cases, ${ }^{21}$ two patients were initially misdiagnosed as optic neuritis. Chiasmal involvement from MOGA can also occur. ${ }^{22}$ In our case, there was no clear radiographic connection between the right frontal lobe lesion and the optic nerve lesion, but the presence of radiographically occult microscopic spread would differentiate multifocal from multicentric GBM.

In summary, although rare, GBM can mimic retrobulbar optic neuritis. Atypical clinical features for ON should prompt neuroimaging. Formal visual field testing of the fellow eye (even if asymptomatic) can lead to the diagnosis of the junctional scotoma. To our knowledge, this is the first reported case in the English language ophthalmic literature of multicentric GBM simulating retrobulbar optic neuritis.

\section{Declaration of interest}

The authors report no conflicts of interest. The authors alone are responsible for the content and writing of the article.

\section{References}

[1] Keltner JL, Johnson CA, Cello KE, Dontchev M, Gal RL, Beck RW. Optic Neuritis Study Group. Visual field profile of optic neuritis: a final follow-up report from the optic neuritis treatment trial from baseline through 15 years. Arch Ophthalmol 2010;128(3):330-7.

[2] Wilbrand H, Saenger A. The neurology of the eyes. $J$ Bergmann 1904;3:98-120.

[3] Wilbrand H. Schema des Verlaufs der Sehnervenfasern durch das Chiasma. Z Aufenheilkd 1926;59:135-144.

[4] Horton JC. Wilbrand's knee of the primate optic chiasm is an artefact of monocular enucleation. Trans Am Ophthalmol Soc 1997;95:579-609.

[5] Shin RK, Qureshi RA, Harris, NR, Bakar D, Li TP, Jafri S, Tang CM. Wilbrand knee. Neurology 2014;82:459460.

[6] Miller NR, Newman NJ, eds. Walsh and Hoyt's Clinical Neuro-Ophthalmology. 5th ed. Vol. 1. Baltimore, MD: Williams and Wilkins; 1998:294-295.

[7] Traquair HM. An Introduction to Clinical Perimetry. St Louis, MO; CV Mosby; 1927:58-77.

[8] Lee AG. Acromegaly and junctional visual field loss. Ophthalmology 2001;108:832-833.

[9] Simao LM, Sultan EMED, Hall JK, Reardon DA, Bhatti TM. Knee deep in the nerve. Surv Ophthalmol 2011;54:362-370.

[10] Pau D, Al Zubidi N, Yalamanchili S, Plant GT, Lee AG. Optic neuritis. Eye 2011;25:833-842.

[11] Hickman SJ, Kupersmith MJ, Straga J, Egan R, Kraker R, Lefton D, Miszkiel KA, Miller DH, Plant GT. Upper temporal visual field depressions in the fellow eye in posterior acute optic neuritis: 'knee' or no 'knee', Wilbrand's concept remains clinically significant. Neuro-Ophthalmology 2002;28:69-76.

[12] Lin C-Y, Huang H-M. Unilateral malignant optic glioma following glioblastoma multiforme in the young: a case report and literature review. BMC Ophthalmology 2017;17:21.

[13] Hoyt WF, Meshel LG, Lessell S, Schatz NJ, Suckling RD. Malignant optic glioma of adulthood. Brain 1973;96:121-132. 
[14] Wabbels B, Demmler A, Seitz J, Woenckhaus M, Bloss HG, Lorenz B. Unilateral adult malignant optic nerve glioma. Graefes Arch Clin Exp Ophthalmol 2004;242:741-748.

[15] Miller NR. Primary tumours of the optic nerve and its sheath. Eye 2004;18:1026-1037.

[16] Pecen PE, Bhatti MT. Clinical reasoning: a 61-year-old woman with a swollen optic nerve and progressive visual loss. Neurology 2014;10;82:e205-e209.

[17] Mattson RH, Peterson EW. Glioblastoma multiforme of the optic nerve. JAMA 1966;196:799-800.

[18] Spoor TC, Kennerdell JS, Martinez AJ, Zorub D. Malignant gliomas of the optic nerve pathways. Am J Ophthalmol 1980;89:284-292.

[19] Balachandran C, Millar MJ, Murali R, Bulliard C, Malcolm CM, Maloof A. Malignant optic glioma presenting as an acute anterior optic neuropathy. Retin Cases Brief Rep 2009;3:156-160

[20] Matloob S, Fan JC, Danesh-Meyer HV. Multifocal malignant optic glioma of adulthood presenting as acute anterior optic neuropathy. J Clin Neurosci 2011;18:974-977.

[21] Traber GL, Pangalu A, Neumann M, Costa J, Weller M, Huna-Baron R, Landau K. Malignant optic glioma -the spectrum of disease in a case series. Graefes Arch Clin Exp Ophthalmol 2015;253:1187-1194.

[22] Lyapichev KA, Bregy A, Cassel A, Handfield C, Velazquez-Vega J, Kay MD, Basil G, Komotar RJ. Glioblastoma multiforme of the optic chiasm: a rare case of common pathology. Surg Neurol Int 2016;7: S485-S487. 